\title{
Power Outage Detection Methods for the Operation of a Shunt Active Power Filter as Energy Backup System
}

\author{
Bruno Exposto, J.G. Pinto, and João L. Afonso \\ Centro Algoritmi, University of Minho, Guimarães, Portugal \\ \{bruno.exposto, gabriel.pinto, \\ joao.1.afonso\}@algoritmi .uminho.pt
}

\begin{abstract}
This paper presents the study of power outage detection methods that can be applied to a Shunt Active Power Filter (SAPF) with energy backup capability. SAPFs can successfully compensate Power Quality problems related with distorted or unbalanced currents and low power factor. Future Smart Grids will combine devices, control strategies and functionalities to increase the grid reliability and the power management capability. One of the main tools necessary to enable these features is the information of what is occurring in all the smart grid parts. In this context the fast detection of power outages is critical, so this paper also contributes for the discussion of the best ways to extract information in the context of future smart grids. The combination of information and flexible devices in a smart grid will enable the implementation of collective awareness systems, which can deal with different electrical grid problems and situations in an organic manner.
\end{abstract}

Keywords: Shunt Active Power Filter, Energy Backup, Power Quality, Power Outage Detection, Kalman Filter.

\section{Introduction}

Smart Grids will become a reality in the future. Efforts are being made to pursue this objective in several countries of the world [1], [2]. The implementation of this new technology implies massive resources deployment, i.e., it must be implemented, study and regulated different parts that will constitute the smart grid. In the technical point of view, Smart Grids generically will integrate three major systems: infrastructure system, smart management system and smart protection system [2]. This technical global vision, although that can change according with the definition of each one point of view and work scope, provides a framework for what can be studied in the Smart Grid context. In terms of infrastructure it must be studied new ways of delivering energy, with the maximum quality, efficiency, reliability and controllability. To achieve these goals, it must be studied new types of power electronics devices, extend the existing power electronics devices capabilities and inherently study new types of control systems.

To achieve good Power Quality indexes, future Smart Grid probably will have to integrate different Power Quality Conditioners [3], [4]. Today are available several 
types of Power Quality Conditioners, namely shunt conditioners [5], series conditioners [4], and the integrated approach known as Unified Power Quality Conditioners (UPQC) [4], [6]. Besides these types of Power Quality Conditioners, exist also in the literature, several references to other devices that have Power Quality compensation capabilities [7].

Shunt Active Power Filters (SAPFs) are shunt connected with the electrical power grid to compensate current problems. This type of Power Quality Conditioners usually is designed to mitigate the current harmonics, the current unbalances, low power factor and the neutral currents [8].

SAPFs are generally constituted by an inverter that can be single-phase or three-phase, voltage-source or current source. There are more defining characteristics that can be applied to the inverters that are used in SAPF, but the focus in this work is three-phase voltage-source inverters [5].

In normal grid operation, the SAPF is compensating the load currents, but when a power outage occurs, the load and the SAPF will be disconnected due to lack of energy. If the process that is being executed by the load is critical, the placement of an SAPF upstream of the load will not useful to deal with the situation of a power outage. In this situation will be necessary to deploy backup equipment like Uninterruptible Power Supplies (UPS). If the power line problems are outages, blackouts or losses of the utility line with time bigger than $10 \mathrm{~ms}$, sags or dips, or even short under-voltages with lasting time less than $16 \mathrm{~ms}$ or even overvoltages with lasting time inferior to $16 \mathrm{~ms}$, the Off-line UPS can be a good solution [9]. Considering that the power converters of a three phase offline UPS is very similar to the SAPF hardware, it would be advantageous combine the two functionalities in the same equipment.

Given this, in this paper is proposed a topology of SAPF that combines the functionalities of current compensation, when connected to the power grid, with the UPS functionalities, to feed the loads that are downstream in case of an power outage.

The combination of the two functions can result in a shorter payback time, so this solution has several advantages.

This paper presents some preliminary results from a $\mathrm{PhD}$ work related with the development of devices to the compensate Power Quality problems. The paper describes the hardware topology of the Shunt Active Power Filter, the control system as well as the energy backup control scheme. Also it describes power outage detection schemes. The behavior of the SAPF with energy backup capability is analyzed, and simulation results are presented.

\section{Relationship to Collective Awareness Systems}

Future Smart Grids will have several features: digitalization, flexibility, intelligence, resilience, sustainability, and customization [10]. Those combined features will allow extending the capabilities of the conventional electrical grids, to levels never thought before. The intelligence of Smart Grids will rely always on data. In fact, to reach collective awareness in a complex system is necessary have large data provided by a network of sensors and devices [11]. The Smart Grid will have these two providers of 
data associated with the capability of change in a real time the behavior of the devices. Given this, the study of methods to obtain information, at the same time that are used devices that have the capability to interact with the grid present several advantages in the future Smart Grids. If we consider that the topology of an off-line UPS has some touching points with the topology of the SAPF, combining the two features will result in a device that is capable of increasing the overall Power Quality at the same time that protects the load of power outages and other aforementioned events.

The collection of data, the increase of Power Quality in the grid and the change of behavior of the devices connected to the grid will be addressed in the PhD work so this is a good contribution to the Smart Grid issue and to the collective awareness systems.

\section{Shunt Active Power Filter with Energy Backup}

The topology of the SAPF is composed by a three-phase four wire shunt converter, with three IGBT legs and a split capacitor in the DC-link (Fig. 1). This topology was chosen because enables the compensation of the three-phases plus the neutral, using only six IGBTs. The main disadvantage is the difficulty in the correct regulation of the DC-link voltages i.e. the difficulty in maintain the voltage of the two capacitors $\left(C_{1}\right.$ and $\left.C_{2}\right)$ equal. The power inverter is connected to the power grid trough three LCR passive output filters to eliminate the switching noise. In the DC-link of the SAPF is connected a reversible DC-DC power converter. This power converter provides an interface with the backup battery pack. When the SAPF is operating in a normal mode, the DC-DC converter charges the battery pack. If a power outage occurs and the energy backup mode is triggered, the DC-DC converter transfers energy from the battery pack to the DC-Link and insures that the voltage in this point is always equal to the predefined reference value.

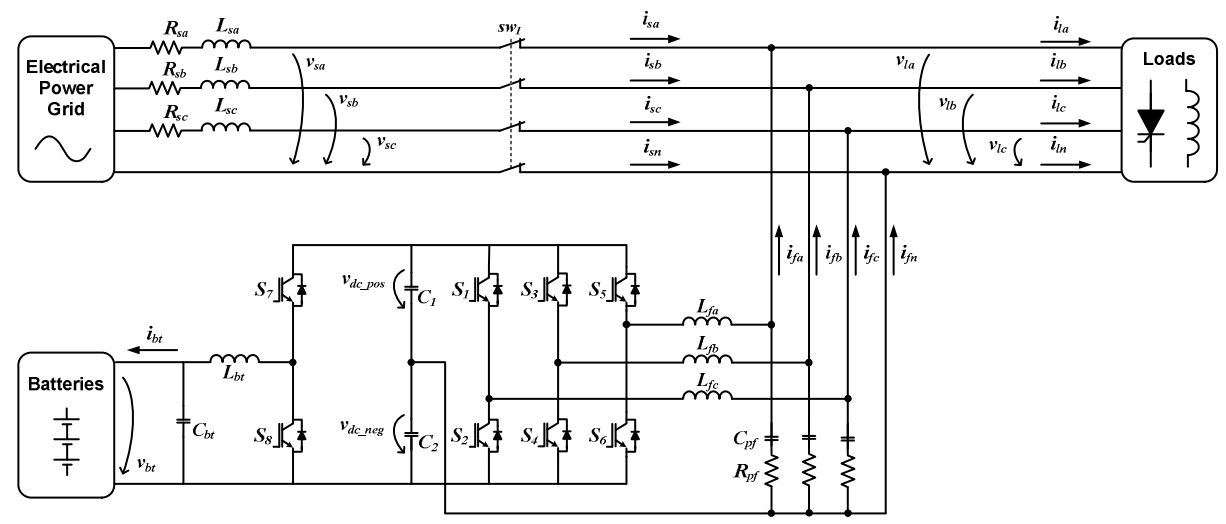

Fig. 1. Topology of the proposed Shunt Active Power Filter with energy backup 
The control of the SAPF is divided in several independent algorithms interacting in an integrated way. To the operation as SAPF, is implemented a control algorithm based in the $p-q$ Theory. Also it was implemented a digital phase locked loop (PLL), that is used in conjunction with the $p-q$ Theory. The detection of the power outage sag or dips is done using a Kalman filter based RMS calculator. The control system is also in charge of the voltage and current control, in both of the operating modes of the $\mathrm{SAPF}$, and the resynchronization after the normal power restoration.

\subsection{Control System}

In the SAPF mode the determination of the compensation currents is done using the $p-q$ theory. This time domain approach has been largely used in SAPFs and Unified Power Quality Conditioners and has the advantages of requiring less processing capability [12], [13].

If the grid voltages are distorted, it is not possible generate compensating reference currents that aim to have sinusoidal source currents, because the $p$ - $q$ Theory will generate reference currents that when injected in the grid and in conjunction with the load currents, will ensure constant power at the source. In this case exists a modification of the $p-q$ Theory that instead of constant instantaneous power at source, results in sinusoidal currents at the source, even with distorted power grid voltages. This modification consists in using the positive sequence of the fundamental voltages instead of the real voltages [13], [14]. To determine the positive sequence of the voltages, in this work was implemented a digital PLL in the $\alpha-\beta$ reference frame. In sum, the use of the digital PLL in conjunction with the control algorithm based in the $p-q$ Theory results in sinusoidal source currents. In Fig. 2 is showed the implementation of the $p-q$ Theory algorithm.

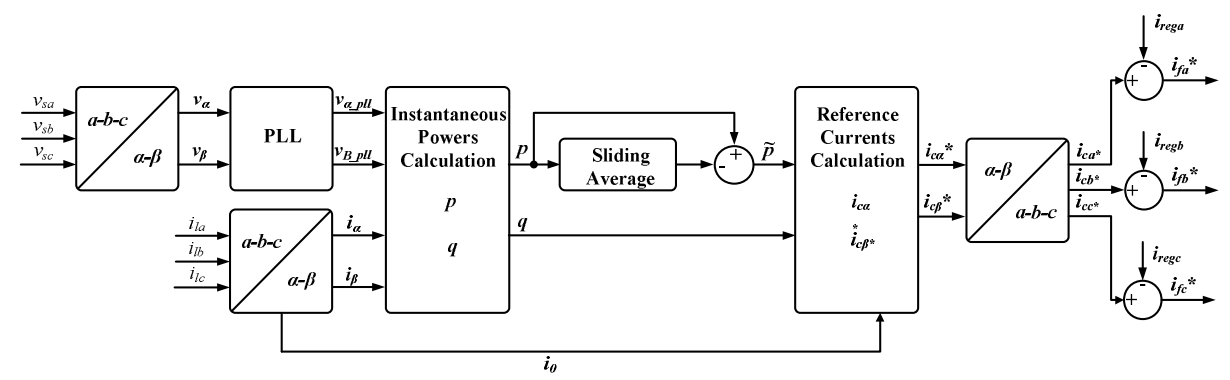

Fig. 2. $p-q$ Theory algorithm implementation

The topology of the inverter composed by a three-phase four wire shunt converter, with three IGBT legs and a split capacitor in the DC-link. In this topology it's necessary that the DC-link capacitors have the same voltage value, or at least an approximated value. To ensure this, is necessary to control these voltages in a separated way. In this work the control of those voltages is done using two (proportional-integral) PI controllers (1). If, for example, $v_{a_{-} p l l} \geq 0$ (calculated through the inverse Clark transform of $v_{\alpha_{-} p l l}$ and $\left.v_{\beta_{-} p l l}\right)$ the top capacitor voltage $\left(V_{D C_{-} p o s}\right)$ is regulated using $i_{\text {rega }}$, 
otherwise is the regulated the bottom capacitor voltage $\left(V_{D C \_n e g}\right)$. This is done for all the three-phases of the SAPF. In (2) and (3) is possible to see how is done this regulation. Finally, the reference compensation current generated by the $p-q$ Theory algorithm $\left(i_{c x}{ }^{*}\right)$ and the regulation current $\left(i_{r x}\right)$ are used to calculate the inverter reference current (3).

$$
\begin{gathered}
p_{r e g_{-} x}=k_{p}\left(V_{D C_{-} x}{ }^{*}-V_{D_{C_{-}} x}\right)+\frac{1}{k_{i}} \int\left(V_{D C_{-} x}{ }^{*}-V_{D C_{-} x}\right) \\
i_{r x}=\left\{\begin{array}{c}
p_{r e g_{-} p o s} \frac{v_{x_{-} p l l}}{v_{a_{-} p l l}{ }^{2}+v_{b_{-} p l l}{ }^{2}+v_{c_{-} p l l}{ }^{2}} \quad, \quad v_{x_{-} p l l} \geq 0 \\
p_{\text {reg_neg }} \frac{v_{x_{-} p l l}}{v_{a_{-} p l l}{ }^{2}+v_{b_{-} p l l}{ }^{2}+v_{c_{-} p l l}^{2}} \quad, \quad v_{x_{-} p l l}<0
\end{array}\right. \\
i_{r e f_{-} x}=i_{c x} * i_{r x}
\end{gathered}
$$

\subsection{Outage Detection Methods}

In this work, the power outage detectors are responsible for evaluate the RMS values of the grid voltages and determine SAPF operation mode. If the system doesn't detect sag, swell or a power outage, the SAPF will operate in the normal mode compensating the load currents. Otherwise, the SAPF will stop the compensation of the loads and operate $n$ backup mode.

The event detection algorithm must be as fast as possible so that the transfer time between normal mode and backup mode be very short. In Moschitta, et al. [15] the authors make a comparison of several sag and power outage detection methods. Their comparison showed that a Kalman filter can be used with success in the detection of sags and power outages decreasing the detection time. The authors propose and discuss other methods but the Kalman filter method has a good performance, is relatively simple to implement and is fast. In this work were implemented three Kalman filters, one per phase. The estimation of the RMS values of the voltages is done using the values obtained in the Kalman filters outputs through the following equation:

$$
V_{R M S}=\sqrt{\frac{\hat{s}[n]^{2}+\hat{q}[n]^{2}}{2}} .
$$

Where $\hat{s}[n]$ and $\hat{q}[n]$ are the estimated values, of voltage and its quadrature respectively. Using the RMS values calculated using the Kalman filters the control system monitors the grid voltages continuously, to determine the operating mode of the SAPF. The control system of the SAPF changes its behavior in a situation where exists a sag higher than $20 \%$ of the nominal voltage, or a power outage. In this situation is activated the energy backup mode of the SAPF and the current control of the inverter is changed to voltage control (the reference voltages are generated using the PLL). Also the loads and the SAPF are isolated from the power grid. 
After the restoration of the normal state of the grid, the control system resynchronizes with the grid and after this, reconnects the load to the grid and returns to the normal mode. In Fig. 3 is possible to see the full operating diagram of the SAPF.

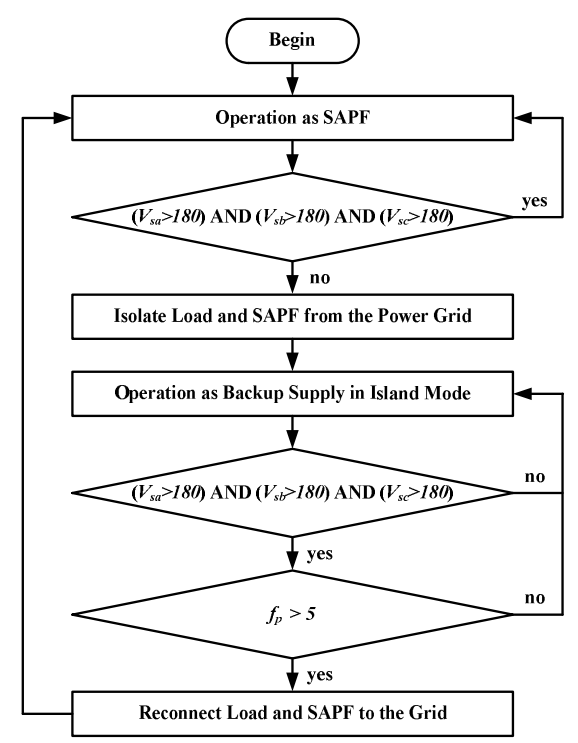

Fig. 3. Shunt Active Power Filter operation diagram

\section{Simulation Models and Simulation Results}

The simulation model is composed by the topology showed in Fig. 1. The simulations were performed using PowerSim PSIM software. In Table 1 is possible to see the simulation parameters values of the model.

Table 1. Shunt Active Power Filter simulation model parameters

\begin{tabular}{ccc}
\hline Parameters & Values & Units \\
\hline$R_{s x}$ & 10 & $\mathrm{~m} \Omega$ \\
$L_{s x}$ & 100 & $\mu \mathrm{H}$ \\
$C_{f x}$ & 25 & $\mu \mathrm{F}$ \\
$R_{f x}$ & 4 & $\Omega$ \\
$C_{x}$ & 10 & $\mathrm{mF}$ \\
$L_{b t}$ & 2 & $\mathrm{mH}$ \\
$C_{b t}$ & 1 & $\mathrm{mF}$ \\
\hline
\end{tabular}

In the simulations, the SAPF starts to compensate the loads (Fig. 4 a) and is possible to see that the source currents $\left(i_{s x}\right)$ are sinusoidal and in phase with the voltages $\left(v_{s x}\right)$. At $\mathrm{t}=0.5 \mathrm{~s}$ the source voltages are removed and then the SAPF acts as energy backup (Fig. 4 b) maintaining the load voltages $\left(v_{l x}\right)$ with the nominal RMS value. The load stays some time being fed by the SAPF for a certain period of time. Then at 
$\mathrm{t}=0.8 \mathrm{~s}$ (Fig. $4 \mathrm{c}$ ) the source voltages are restored and the SAPF starts the resynchronization of the load voltages $\left(v_{l x}\right)$ and the source voltages $\left(v_{s x}\right)$. When the resynchronization is complete $(t=0.815 \mathrm{~s})$, the load and the SAPF is reconnected to the grid and the SAPF returns to normal mode.

(a)

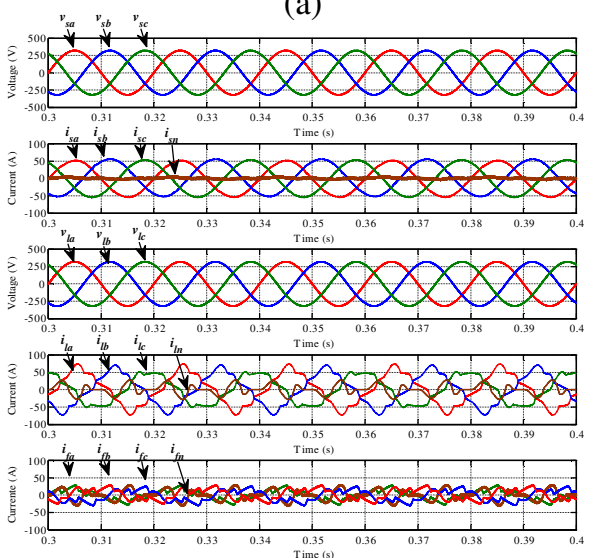

(b)
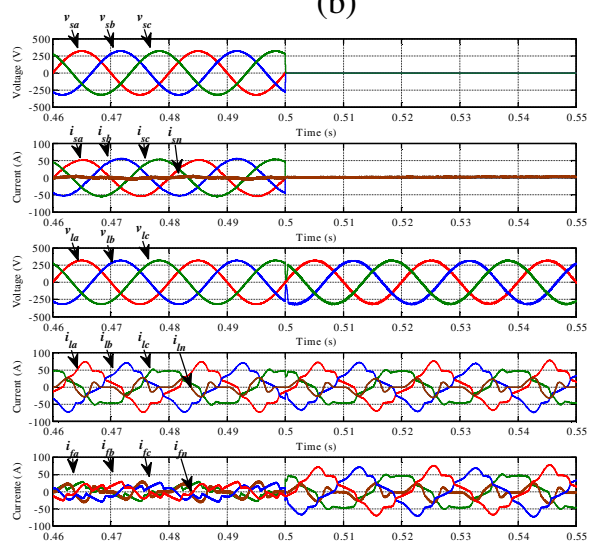
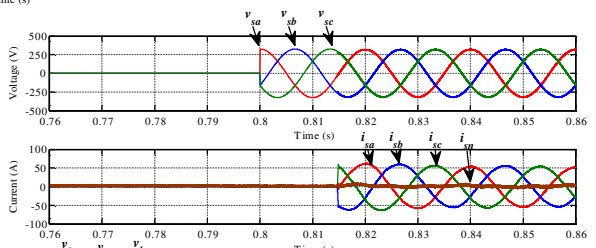

(c)
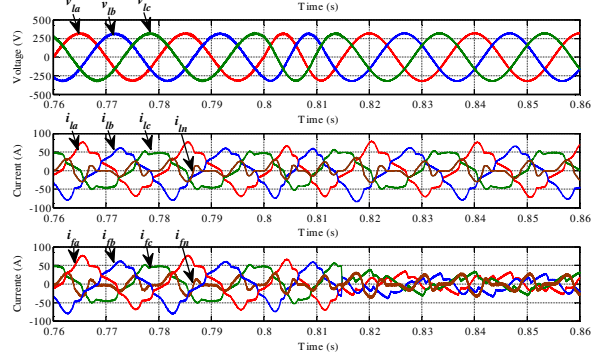

Fig. 4. Simulation results of the SAPF: (a) Operating in normal mode; (b) Operating in backup mode; (c) During transference to backup mode; (d) During transference to normal SAPF mode

\section{$5 \quad$ Conclusions and Future Work}

This paper presents a Shunt Active Power Filter (SAPF) that is able to compensate Power Quality problems and that also serves as energy backup system during power outages. The papers also discuss some methods of power outage detection. The simulation results show that the SAPF can successfully compensate the current harmonics, the current unbalances and the power factor. The simulation results also show that the SAPF can be used as energy backup system in case of a power outage. Also the power 
presented outage detection scheme performs correctly. Nevertheless further tests are needed, namely with different loads and power outage conditions.

Acknowledgment. This work is financed by FEDER Funds, through the Operational Programme for Competitiveness Factors - COMPETE, and by National Funds through FCT - Foundation for Science and Technology of Portugal, under the projects FCOMP-01-0124-FEDER-022674 and PTDC/EEA-EEL/104569/2008. Bruno Exposto is supported by the doctoral scholarship SFRH/BD/87999/2012 granted by FCT - Foundation for Science and Technology of Portugal.

\section{References}

1. Platform, A.C. of the E.T.: SmartGrids - Strategic Deployment Document for Europe's Electricity Networks of the Future (2010)

2. Fang, X., Misra, S., Xue, G., Yang, D.: Smart Grid, The New and Improved Power Grid: A Survey. IEEE Communications Surveys Tutorials 14, 944-980 (2012)

3. Pinto, J.G., Couto, C., Afonso, J.L.: Analysis of the Features of a UPQC to Improve Power Quality in Smart Grids. In: Camarinha-Matos, L.M., Tomic, S., Graça, P. (eds.) DoCEIS 2013. IFIP AICT, vol. 394, pp. 299-306. Springer, Heidelberg (2013)

4. Javadi, A., Geiss, N., Blanchette, H.F., Al-Haddad, K.: Series active conditionners for reliable Smart grid: A comprehensive review. In: IECON 2012 - 38th Annual Conference on IEEE Industrial Electronics Society, pp. 6320-6327 (2012)

5. Singh, B., Al-Haddad, K., Chandra, A.: A review of active filters for power quality improvement (1999)

6. Fujita, H., Akagi, H.: The unified power quality conditioner: the integration of series and shunt-active filters (1998)

7. Reddy, K.N., Agarwal, V.: Utility-Interactive Hybrid Distributed Generation Scheme With Compensation Feature. IEEE Trans. on Energy Conversion 22, 666-673 (2007)

8. Exposto, B., Gonçalves, H., Pinto, J., Afonso, J.L., Couto, C.: Three Phase Four Wire Shunt Active Power Filter from Theory to Industrial Facility Tests. In: 2011 11th International Conference on Electrical Power Quality and Utilisation (EPQU), pp. 1-5 (2011)

9. Gurrero, J.M., de Vicuna, L.G., Uceda, J.: Uninterruptible power supply systems provide protection. IEEE Industrial Electronics Magazine 1, 28-38 (2007)

10. Jiang, Z., Li, F., Qiao, W., Sun, H., Wan, H., Wang, J., Xia, Y., Xu, Z., Zhang, P.: A vision of smart transmission grids. In: Power Energy Society General Meeting, PES 2009, pp. 1-10. IEEE (2009)

11. Pitt, J., Bourazeri, A., Nowak, A., Roszczynska-Kurasinska, M., Rychwalska, A., Santiago, I.R., Sanchez, M.L., Florea, M., Sanduleac, M.: Transforming Big Data into Collective Awareness. Computer 46, 40-45 (2013)

12. Akagi, H., Watanabe, E.H., Aredes, M.: Instantaneous power theory and applications to power conditioning. Wiley-IEEE Press (2007)

13. Afonso, J.L., Couto, C., Martins, J.S.: Active filters with control based on the pq theory (2000)

14. Monteiro, L.F.C., Afonso, J.L., Pinto, J.G., Watanabe, E., Aredes, M., Akagi, H.: Compensation algorithms based on the pq and CPC theories for switching compensators in mi-crogrids. In: Power Electronics Conference, COBEP 2009, Brazilian, pp. 32-40 (2009)

15. Moschitta, A., Carbone, P., Muscas, C.: Performance Comparison of Advanced Techniques for Voltage Dip Detection. IEEE Transactions on Instrumentation and Measurement 61, 1494-1502 (2012) 